\title{
MD-TDOF-PID Controller Based on LabView
}

\author{
Yu Jian, Liu Changliang \\ North China Electric Power University, Baoding, China \\ myset52259@163.com
}

\begin{abstract}
Keywords: two-degree-of-freedom PID,PD feedback compensator,LabView,
\end{abstract}
\begin{abstract}
In this paper,Aiming at the weakness that the commonly used traditional PID controller can not obtain good anti-interference ability and set value following performance, a two degree of freedom model driven PID controller (MD-TDOF-PID) based on Labview is designed. Proportional derivative compensator of the MD-TDOF-PID can be used to transform the the actual controlled production process such as large inertia and large delay system in industrial production or the object difficultly to control with unstable characteristics in open loop system into a first order equivalent controlled object with time delay, and the time constant of the equivalent object is much smaller than that of the actual controlled process. The simulation results show that compared with the traditional PID control and fuzzy variable parameter PID controller the MD-TDOF-PID controller has fast tracking ability and stronger robustness so that it can effectively improve the control performance of the system.Being based on the LabVIEW makes the interface more intuitive and field oriented, and thevisa interface makes communication more compatible, and controller system can be directly applied to the scenewith simple structure and good real-time performance control.
\end{abstract}

\section{Introduction}

In the field of industrial automation, the earliest and most widely used control method is PID control which control theory is the most mature. Because of simple structure, easy tuning parameters, good static performance and high reliability in practical application, conventional PID control has been widely used in industry and other fields since it appeared. The PID control system is still the most basic and widely used technology in the industrial application, however in some controlled process, its control performance has some disadvantages, such as large delay, delay, instability, oscillation and so on.In order to improve the control performance of such controlled object, control field workers have applied the control schemes with advanced control theory such as Smith predictor, internal model control, auto disturbance rejection control and so on, which provide a large number of research results on performance analysis、 stability and robustness of system and the simulation results show that they are effective. Production site needs control system with simple structure, easy adjustment of parameters and good real-time performance. What's more, the system can still meet the requirements of the control performance of the production process in the case of a certain range of process parameters, and is easy to be added to the field DCS configuration (easy to be implemented).

As one of the advanced control algorithm, internal model control has drawn more and more attention of researchers since it was proposed in. A model driven control(MDC)concept was proposed by Tsumura and Kimura as an alternative control system of IMC.in 2002 ,A model driven PID control system(MD PID) developed by Masanori combines an MDC control system with a PD local feedback, an IMC , and a set point filter.in[1], Yukitomo et al. suggested using a model driven Two-Degree-of-Freedom PID(MD-TDOF-PID) to replace the IMC which has One-Degree-of-Freedom, with an IMC Q-filter which has Two-Degrees-of-Freedom to have a better control effect.

Paper design a MD-TDOF-PID controller based on LabVIEW program, for industrial communication system is compatible with the most way of communication. Comparing to other advanced control algorithm, MD-TDOF-PID control system has simple structure, good real-time performance and control parameter is easy to adjust and does not need special complex theory, 
convenient for application in the field. Based on the LabVIEW can make more intuitive interface and oriented to the scene, and the LabVIEW VISA interface can make communication more compatibility.

\section{2. structure of model-driven two-degree-of-freedom PID control system}

\subsection{MD-TDOF-PID control system configuration}

MD-TDOF-PID control system was composed of PD feedback compensator,main controller and set point filter,and the main controller can be further decomposed into a gain block,a second order $\mathrm{Q}$ filter with tuning parameter $\lambda, \alpha$ and a first order model with time delay,shown in Fig.1.which $r, r_{y}, e, v, u, y$ was setpoint,output from setpoint filter,error,output from main controler,controler output and process variable.

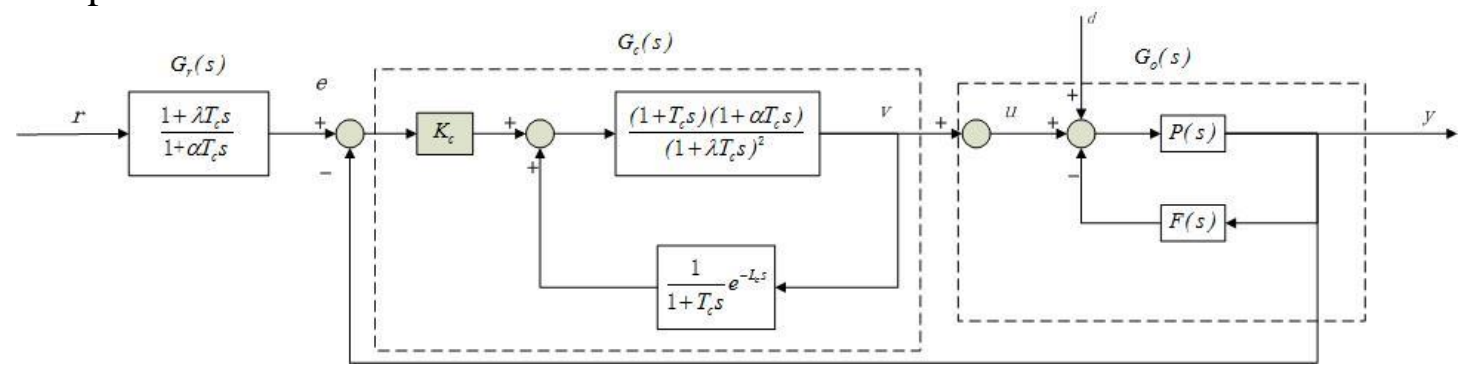

Fig1.MD-TDOF-PID control system

\subsection{PD feedback compensator $F(s)$}

PD feedback compensator is used to improve the system dynamic characteristics for stable system, and for unstable system PD feedback $F(s)$ can made system stable.

PD feedback compensator including the PD feedback itself $F(s)$ make the transfer function of the process to a first order system with time delay[1].The transfer function of the controlled system from $v$ to $y$ is expressed by:

$$
G(s)=\frac{P(s)}{1+P(s) F(s)}=\frac{K^{-L s}}{1+T s}
$$

where $K, T$ and $L$ are gain,time constant and dead time of the overall controlled process respectively.Well designed PD feedback conmpensator would get control parameters ,gain $K$ time constant $T$ and dead time $L$ properly, which the integral error of MD-TDOF-PID control system will be smaller than that of the originally controlled process.

When $P(s)$ is identified ,the PD feedback $F(s)$ can be designed by using many design methods such as the principle pole place allocation method, gain margin and phase margin method,the root locus method,model match method and optimization method the PD feedback compensator was designed using model matching method in [2],the PD feedback compensator is designed as:

$$
F(s)=\frac{K_{f}\left(1+T_{f} s\right)}{1+\gamma T_{f} s}
$$

where $K_{f}, T_{f}$ and $k$ are constants.

So long time delay process, oscillation process and unstable process can be controlled by MD-TDOF-PID controller.

\subsection{Main controller $C(s)$}

The main controller consists of three blocks: gain block $K_{c}, \mathrm{Q}$ fliter $Q(\mathrm{~s})$ and ideal model $G(s)$, which compensated by PD feedback.After $G(s)$ is identified in the form of Eq.(1).the main controller is designed by adjusting the parameters $\lambda$ and $\alpha$ in $Q$ filter to meet the design specifications. The main controller transfer function is derived as follows.

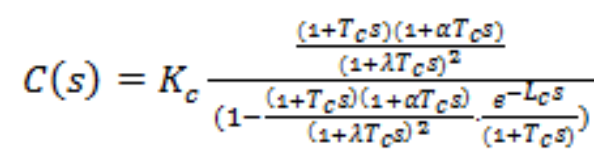


where $K_{c}$ is controller gain and $T_{c}$ is controller time constant.

The open-loop transfer function of system(from $e$ to $y$ )is

$$
G_{1}(s)=\frac{1}{K} \frac{(1+T s)(1+\alpha T s)}{(1+\lambda T s)^{2}-(1+\alpha T s) e^{-L s}} \cdot \frac{K e^{-L s}}{(1+T s)}=\frac{(1+\alpha T s) e^{-L s}}{(1+\lambda T s)^{2}-(1+\alpha T s) e^{-L s}}
$$

the closed-loop transfer function of system(from $r_{y}$ to $y$ )is derived as

$$
G_{0}=\frac{G_{1}}{1+G_{1}}=\frac{(1+\alpha T s) e^{-L s}}{(1+\lambda T s)^{2}}
$$

the transfer function from $d$ to $y$ is

$$
\frac{y}{d}=\frac{G(s)}{1+G_{\mathrm{I}}(s)}=\frac{\frac{K e^{-L s}}{(1+T s)}}{1+\frac{(1+\alpha T s) e^{-L S}}{(1+\lambda T s)^{2}-(1+\alpha T s) e^{-L s}}}=\frac{K e^{-L S}}{(1+T s)}\left[1-\frac{(1+\alpha T s) e^{-L s}}{(1+\lambda T s)^{2}}\right]
$$

The main controller improves the speed response ability of the system.In the main controller, $K_{c}$ is used to cancel $K$ in $G(s)$ to make sure that $G_{0}(0)=0$,thus the steady state position error is zero i.e. ep $=0$ for step input signal $(r)$. The term $(1+\mathrm{Ts})$ is used to cancel the term $(1+\mathrm{Ts})^{-1}$ in the $G(s)$ by setting $T_{c}=$ T.It is very effective in improving the speed response ability of system . In Eq.(1),the time delay item can be approximated as $\mathrm{e}^{-L s} \approx \frac{1}{1+L s}$ (in low frequency region), hence one has

$$
G(s) \approx \frac{K}{(1+T s)(1+L s)}
$$

If the slow pole $\left(\mathrm{s}=-\frac{1}{T}\right)$ is removed, $G(s)$ will become much faster than the previous one. The main controller helps in rejecting the noise $d$ from entering system .From Eq.(7), in low frequency region $\left(s \approx 0\right.$ ), one has $\frac{y}{d} \approx 0$ which means the noise is rejected from entering system.It is noticed that if the pole is $s \approx 0$, one has $\frac{y}{d} \approx 1$.

Hence,the main controller can allow almost all reference signals enter into system while the noise is rejected.

\subsection{Set point filter}

The set point filter is used to cancel one zero and one pole in the system and thus to reduce the order of the system from second order to first one.

$$
\frac{y}{r}=\frac{(1+\lambda T s)}{(1+\alpha T s)} \frac{(1+\alpha T s) e^{-L s}}{(1+\lambda T s)^{2}}=\frac{e^{-L s}}{(1+\lambda T s)}
$$

\section{MD-TDOF-PID Control system design and LabView Program build}

LabView is short for laboratory virtual instrument engineering workbench. It was launched by the U.S. national instrument company, mainly oriented to virtual instrument in the field of computer measurement and control software development platform. It is a integrated environment which is based on graphic development, debugging and running.

The data processing VI serves as the main-VI, while the communication VI serves as the sub-VI, which is called by the main-VI, its data transferred to the main-VI via the global variable. The main function of the data processing part is PID operation and human-computer interface. Its front panel refer to figure. 2 . 


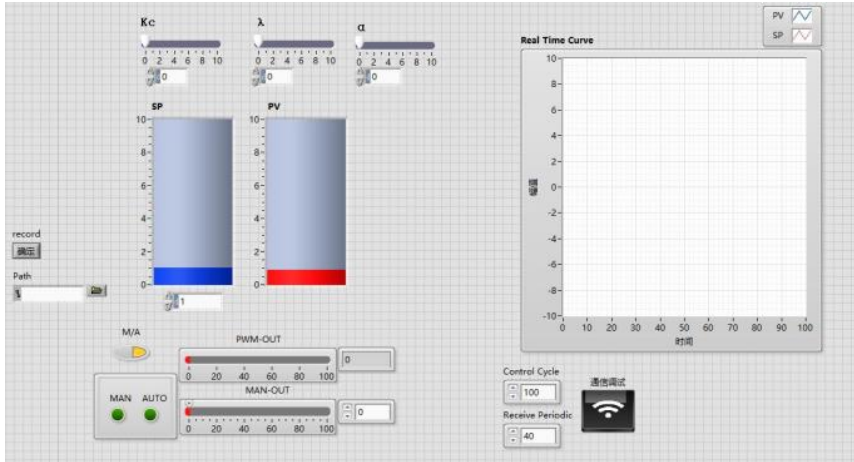

Fig2. The front panel of MD-TDOF-PID control system based on LabView

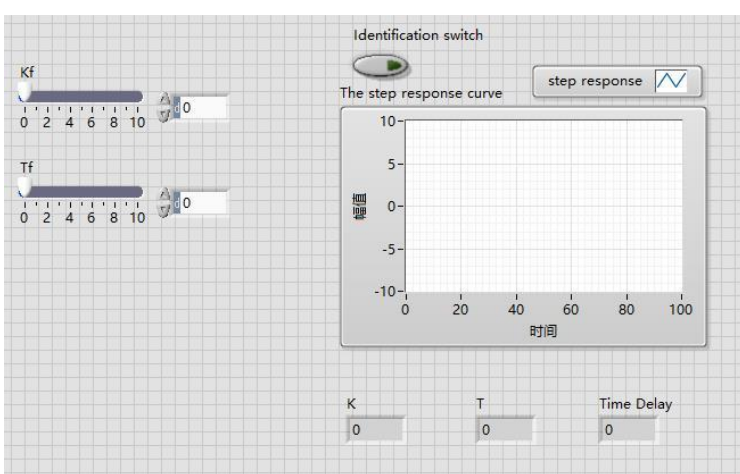

Fig3. PD feedback compensator based on LabView

\subsection{PD feedback compensator design}

As a separate part of the system, the effect of PD feedback compensator is to improve the control quality of controlled object, In the case of $G(s)$ is a long time-delayed system, MD-TDOF-PID main controller is not better than the traditional PID controller in control quality, so the PD feedback compensator is the way to improve the dynamic characteristics of objects, to make the controller to achieve better control effect. There are a lot of PD feedback compensator design method, the program provides a setting module for $F(s)$, Shown in figs 3 。

Users can choose according to use the method to calculate the corresponding system $F(s)$, and then to set parameters to adjust the dynamic characteristics of the closed-loop system $G(s)$.

\subsection{Main controller design}

PD feedback compensator can converte control object into a first order system with time delay.by expanding $e^{-L s}$ in $G(s)$ into Maclaurin series in $s=0$,one has:

$$
G(s)=\frac{K}{1+(T+L) s+\left(T L+\frac{L^{2}}{2}\right) s^{2}+\left(T L^{2}+\frac{L^{s}}{6}\right) s^{s}+\cdots}
$$

When the controlled object is stable and no overshoot amount of ideal, the ideal object transfer function can be expressed as:

$$
G(s)^{\prime}=\frac{1}{\left(f_{0}+P_{0}\right)\left[1+\sigma s+\beta_{2}(\sigma s)^{2}+\beta_{g}(\sigma s)^{\mathrm{s}}\right]}
$$

Matching $G(s)^{t}$ and $\mathrm{G}(\mathrm{s})$ :

$$
\left\{\begin{array}{c}
K=1 /\left(f_{o}+p_{0}\right) \\
T+L=\sigma
\end{array}\right.
$$

setting:

$$
\mathrm{L}=\mu \mathrm{T}
$$

Following equations(11) can be derived from eq.(10) and eq.(11):

$$
\mathrm{T}=\sigma /(1+\mu)
$$

radio $\mu$ can be calculated as followed.

Due to coefficient of $s^{2}$ is equal can get $\mathrm{TL}+\frac{L^{2}}{2}=\beta_{2} \sigma^{2}$, and

$$
\left\{\begin{array}{c}
L=\mu T \\
T=\sigma /(1+\mu)
\end{array}\right.
$$

where the radio $\mu=1.0412$ by solving equation:

$$
\frac{1+\mu^{2} / 2}{(1+\mu)^{2}}=\beta_{2}
$$

The parameters of the controller $C(s)$ can calculated by eq.(9)-(15) as $K_{c}=\frac{1}{K}, T_{c}=T_{s} L_{c}=L$. 
The main proportion controller is divided into several proportional component, first order component and time-delay component, set the parameters as $K_{c}=\frac{1}{K}, T_{c}=T_{s} L_{c}=L$, user can adjust the control performance of the main controller by adjusting the value of $K_{c}$.

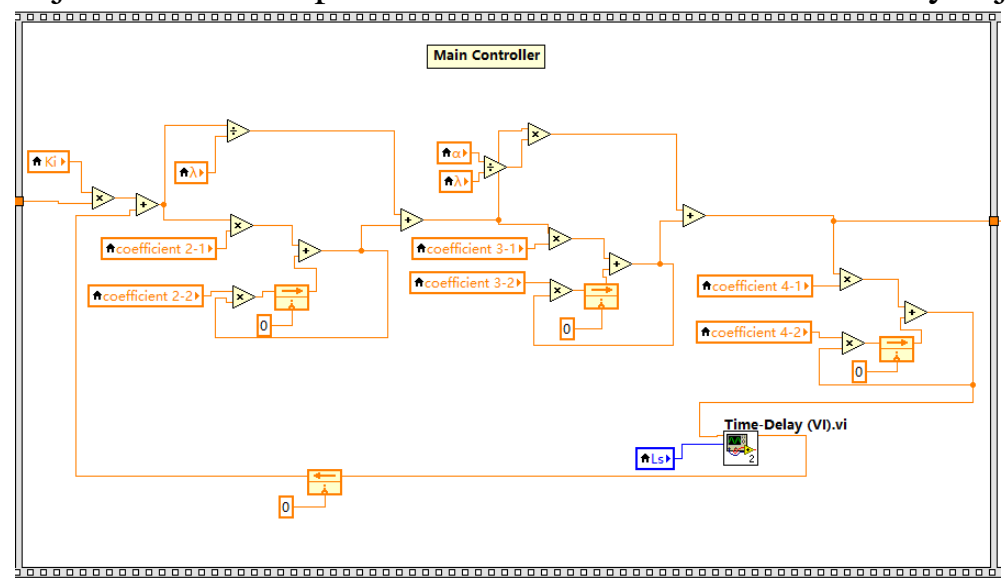

Fig.4. Main controller design on LabView

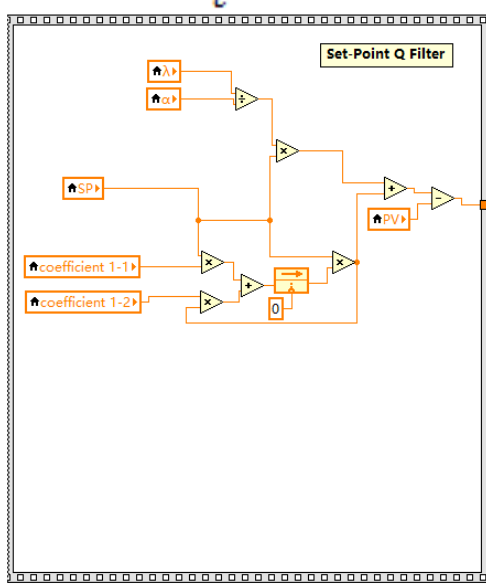

Fig.5. Q-fliter design on LabView

\subsection{Set-point $Q$ filter design}

Adjust the set value filter parameter is determined by setting the parameters $\lambda$ and $\alpha$,there are many ways like theoretical calculation method and experience method, etc can calculate the value of its. General common design formula is as follows:

$$
\left\{\begin{array}{c}
\lambda=\frac{1}{K u_{\lim }} \lim _{s \rightarrow 0}\left[\left(1+P(s) F(s)^{-1}\right)\right] \\
\alpha=\frac{L}{T}\left(1-\lambda^{2} T^{2} \omega^{2}\right)+2 \lambda\left(1-L^{2} \omega^{2} / 2\right)
\end{array}\right.
$$

$u_{\lim }$ is the allowed maximum of operating variables $u, \omega$ is the disturbing frequency.

For some higher-order system, theoretical calculation parameter is not the best due to its mathematical model is not enough accurate, therer also need to optimize the parameters adjustment.Adjust the parameters value of $\lambda$ and $\alpha$ can improve the control performance of system and improve the anti-interference ability, and the traditional PID cannot meet the two characteristics at same time, value of $\lambda$ and $\alpha$ are general between 0 and 1 .

\section{Simulation}

MD-TDOF-PID is better than tranditional PID controller in control quality for time delay inertial system. Simulation objects, oxygen measuring instrument of Zirconia, consists of a heating furnace and a thermocouple, conducted by the furnace temperature control, to the realization of thermocouple temperature feedback control for reheating furnace of duty ratio

The simulation object selects temperature control system for oxygen measuring instrument of Zirconia. The system consists of two parts. One is a heating furnace, which controls the temperature of system. The other is thermocouple, feeding back the values of real-time temperature. And control amount of the system is duty ratio of heating furnace. Meanwhile, the mathematical model for furnace can be approximately described as a first-order inertia with pure lags and the one for thermocouple is basically a great inertia and long delay system.

The transfer function of object is $P(s)=\frac{1.1}{120 s+1} e^{-40 s}$ by system identification.

PD feedback compensator is designed based on model matching method.Due to eq.10 when $\beta_{2}=0.38, \beta_{3}=0.08$ response curve with no overshoot,calculated $F(s)=\frac{10.8886 s+1.0672}{0.5101 s+1}$.

$$
G(s)=\frac{P(s)}{1+P(s) F(s)} \approx \frac{K e^{-L s}}{1+T s}=\frac{0.506}{40.3358 s+1} e^{-41.9976}
$$

According to the formula of setting up the controller parameters: $\alpha=0.5, \lambda=1, \mathrm{~K}=2.25$. 
And setting the tranditional PID controller parameters as: $K_{p}=1.1, K_{i}=0.018, K_{d}=0$ by attenuation curve method.

Value as a unit step change when $\mathrm{t}=1 \mathrm{~s}$. The results as shown fig. 6

Add disturbance under normal working state, observing the ability to resist disturbance of the controller, shown as fig.7

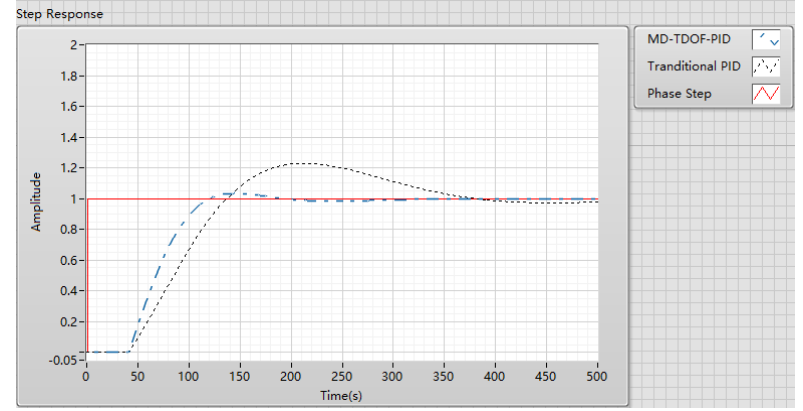

Fig.6. The response curve of the step signal

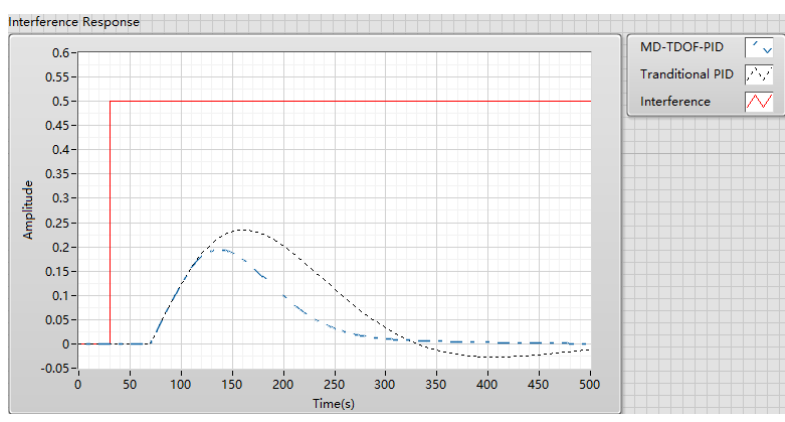

Fig.7. The response curve of the interference

Fig.6 shown that inn the same circumstances, MD-TDOF-PID overshoot volume smaller, and the adjusting time is short, response faster, and has better set point tracking ability.Besides,fig.7 shown MD-TDOF-PID also has Anti-interference ability. The simulation results show that the controller can be put into use after parameter setting and has excellent control quality.

\section{Conclusion}

The paper design a MD-TDOF-PID controller based on LabView, and simulate in oxygen measuring instrument of Zirconia. Compare with tranditional PID controller ,MD-TDOF-PID has less overshoot, and short settling time. Beside, MD-TDOF-PID controller based in Labview has simple structure is easy to adjust parameters, and can widely used in industrial process control to improve the control performance of controlled object.

\section{References}

[1] Yukitomo M, Shigemasa T, Baba Y, et al. A two degrees of freedom PID control system, its features and applications[C]//Control Conference, 2004. 5th Asian. IEEE, 2004, 1: 456-459.

[2] Shigemasa T, Yukitomo M, Kuwata R. A model-driven PID control system and its case studies[C]//Control Applications, 2002. Proceedings of the 2002 International Conference on. IEEE, 2002, 1: 571-576.

[3]Baba Y,Shigemasa T, Yukitomo M,et al,Model-driven PID control system in single-loop controller[C],Sice Conference.2003,1,197-190.

[4]Shigemasa T, Y. lino and M. Kanda, Two Degrees of Freedom PID Auto-tuning Controller[C],87-1191,Proceedings of the ISA,Anaheim,1987.

[5]Shinskey F.G,Feedback Controllers for the Process Industries[M],McGraw-hill,1994

[6] Fakhrulddin H. Ali, Mohammed Mahmood Hussein, Sinan M.B. Ismael, LabVIEW FPGA Implementation Of a PID Controller For D.C. Motor Speed Control[C], 2010 1st International Conference on Energy, Power and Control (EPC-IQ),2010.10,139-144. 